\title{
PAPER \\ Harmonic Reduction and Chaotic Operation towards Application of AC/AC Converter with Feedback Control
}

\author{
Alexandros KORDONIS ${ }^{\dagger a}$, Nonmember and Takashi HIKIHARA ${ }^{\dagger}$, Fellow
}

\begin{abstract}
SUMMARY AC conversion has a huge variety of applications and so there are many ongoing research topics as in every type of power electronic conversion. New semiconductors allow the increase of the switching frequency fact that brings a whole new prospective improvement in converter's operation. Many other possible nonlinear operation regimes, including period doubling and chaotic oscillations, appear besides the conventional steady state operation. In this work, a nonlinear discrete-time model of an AC/AC buck type converter is proposed. A discrete time iterative map is derived to highlight the sensitive switching dynamics. The model is able to observe fast scale phenomena and short transient effects. It offers more information compared to other methods such as the averaging ones. According to Electro-Magnetic Compatibility (EMC) regulations, low wide-band noise is more acceptable than the high narrowband, therefore the goal of this work is to spread the harmonic noise into a wide frequency spectrum which has lower amplitudes compared to the conventional comb-like spectrum with distinctive amplitudes at switching frequency multiples. Through the numerical and experimental consideration the converter can operate in a chaotic motion and the advantages of the performance improvement are also discussed.

key words: AC/AC converter, nonlinear dynamics, bifurcation, chaos, feedback control, harmonics, EMC, iterative map
\end{abstract}

\section{Introduction}

The developments and achievements of nonlinear theory in the power electronics field raise the interest and the possibility of a new era of power converters [1]. For many years researchers have also dealt with the problem of harmonic elimination as for example in [2]-[5]. In particular much research has been done, covering in depth many operations of the so called PWM AC voltage controller. It is already known that this type of converter has superior characteristics compared to the lower quality phase-controlled one [6]. It can be used in static ac switches, soft starters, windturbine systems, lighting control, industrial heating systems, compensation of unbalanced supplies, and so on. From the viewpoint of applications, it is obvious that its research field becomes wider and hasn't yet been fully investigated.

When designing a converter, the switching frequency, semiconductor choice, and control design must be well adjusted [7]-[10]. In this report, the feedback gain and switching frequency are focused for discussion. Previous research has already discussed the possibility of variable gain control methods pointing the advantages such as faster transient response, better regulation characteristics, and so on [11],

Manuscript received June 14, 2013.

Manuscript revised October 3, 2013.

${ }^{\dagger}$ The authors are with the Department of Electrical Engineering, Kyoto University, Kyoto-shi, 615-8510 Japan.

a) E-mail: kordonis@dove.kuee.kyoto-u.ac.jp

DOI: $10.1587 /$ transfun.E97.A.840
[12]. In the last decades the wide-spread frequency spectrums have been explored at specific operational regimes of system with switching, for example when it operates chaotically [13]. The power converter also has a possibility of the noise reduction by this method. Such behavior brings results with better EMC (Electro-Magnetic Compatibility) and improved agility [14], [15]. The EMC regulations request wide-band noise than narrow-band. Therefore it is of great importance if, for example, the switching harmonics could be spread in a wide frequency range with small amplitudes rather than having distinctive high peaks.

In this paper, the iterative map derivation along with the control description gives the formula for the converter discrete-time model. By deriving this model we are exploiting the advantages of this method in order to show the behavior of switching dynamics for an AC/AC converter implemented with bidirectional switches, LC filter and load. Moreover, all the nonlinearities such as period doubling and chaotic operation are described through bifurcation diagrams and Lyapunov exponents. Last but not least, the experimental approach and a discussion about it will support the validity of the mathematical model and will show the potential for application realisation. Including this introduction, this work consists of six sections. Section 2 describes in brief the iterative map derivation and the control technique. In Sect. 3, the switching frequency and feedback gain impact are analyzed and in the following Sect. 4, the chaotic confirmation analysis is presented. Section 5 is the presentation of the experimental results and a small discussion based on these ones. Finally, the last section concludes this report by summarizing.

\section{Iterative Map and Control Description}

The AC/AC converter requests the current to flow in both directions, therefore bidirectional switches are used. In experiments, two power Mosfets with their sources connected form a bidirectional switch. In the simulation, ideal switches have been used without loss of generality. This paper introduces a nonlinear model with difference equations in order to solve its dynamics. The basic idea is to solve the differential equations of the converter using a discrete time iterative map. The converter under investigation is presented in Fig. 1 and obeys the unified differential equation system of Eq. (1). The dynamic system state variables are the capacitor $(C)$ voltage and inductance $(L)$ current. $E$ is the input voltage of the circuit and $R$ represents the load. The switch- 


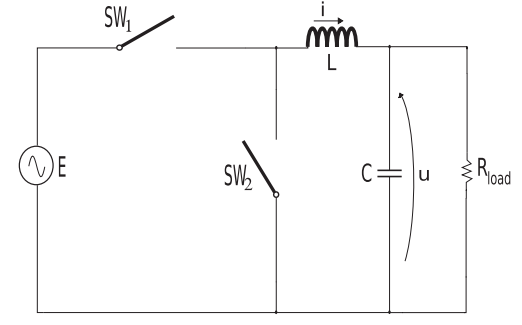

Fig. 1 AC/AC converter topology with ideal bidirectional switches.

ing variable $s$ represents the on state at $s=1$ and off state at $s=0$, respectively. The on state represents the time duration when the source is feeding the load with power and the off state when the freewheeling current flows through $\mathrm{SW}_{2}$. Based on the iterative map, the behavior from one to the next switching cycle can be described.

$$
\begin{aligned}
& \left\{\begin{array}{c}
L \frac{\mathrm{d} i}{\mathrm{~d} t}=s E-u \\
C \frac{\mathrm{d} u}{\mathrm{~d} t}=i-\frac{u}{R}
\end{array}\right. \\
& i_{1}(t)=f\left(u\left(t_{n}\right), i\left(t_{n}\right),\left(t-t_{n}\right)\right) \\
& i_{2}(t)=g\left(u\left(t_{n^{\prime}}\right), i\left(t_{n^{\prime}}\right),\left(t-t_{n^{\prime}}\right)\right) \\
& d_{n}=D-k\left(u_{n}-V_{r e f}\right)
\end{aligned}
$$

A complete switching period happens every $\left(t_{n}, t_{n+1}\right)$, where $n \in \mathbb{N}^{+}$. Let $t_{n^{\prime}}$ be the arbitrary moment the switching happens. Assume without harming the generality of the problem that $\mathrm{SW}_{1}$ is off and $\mathrm{SW}_{2}$ is on for $t_{n}<t<t_{n^{\prime}}$ and vice versa for the rest of the switching period $\left(t_{n^{\prime}}<t<t_{n+1}\right)$. Solving the differential equation when $s=0$ for the $\left[t_{n}, t_{n^{\prime}}\right)$ interval Eq. (2) is derived. The solution during the interval $\left[t_{n^{\prime}}, t_{n+1}\right)$ has the form of Eq. (3). The same procedure is followed for the other state variable of the problem. Therefore, the following description is devoted only to one state variable, the inductance current.

The key point here is to achieve one and only relation by using Eqs. (2) and (3). This relation has to be independent from the arbitrary switching time $t_{n^{\prime}}$, and the continuous time $t$. Therefore, wherever there is $t$ in Eq. (3) is replaced by $t_{n+1}$, and the difference $t_{n+1}-t_{n^{\prime}}$ is equal to $d T$, where $d$ denotes the duty cycle and $T$ the switching period. At this moment, $i\left(t_{n+1}\right)$ is described from the duty cycle $d$ and the values of $i\left(t_{n^{\prime}}\right)$ and $u\left(t_{n^{\prime}}\right)$. These two values can now be found using Eq. (2) by replacing $t$ with $t_{n^{\prime}}$. The difference $t_{n^{\prime}}-t_{n}$ is equal to $(1-d) T$. Thus the formula which describes the next value of current with the previous one without the interference of the arbitrary switching moment is finally succeeded. In other words, a difference equation is derived of the form $\boldsymbol{x}_{n+1}=q\left(\boldsymbol{x}_{n}, d_{n}\right)$, where $\boldsymbol{x}$ is the vector of the state variables and $d_{n}$ is governed by Eq. (4). It can be illustrated by the proportional feedback control layout at Fig. 2. $D$ denotes the steady state duty cycle, $k$ the feedback gain, $V_{\text {ref }}$ the reference voltage, and $d_{n}$ the (instant) duty cycle. The actual instant duty cycle $\left(d_{n}\right)$ is variable. According to the difference between the output and reference voltage,

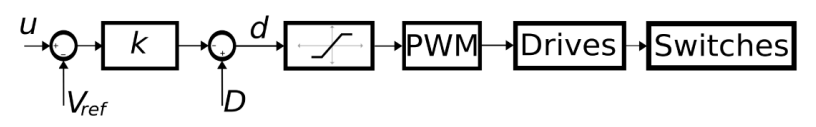

Fig. 2 Control configuration of feedback regulator.

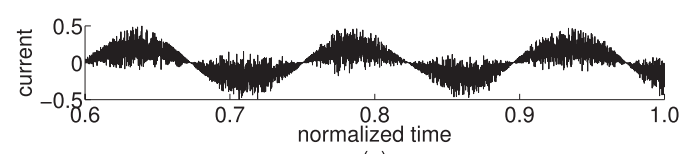

(a)

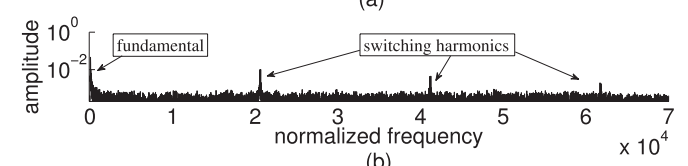

(b)

Fig. 3 Current in steady state (a) and its frequency spectrum (b). Current contains the fundamental, switching harmonics and switching noise.

in every switching cycle, the duty cycle is decided.

It should be noted that the duty cycle function is limited between $[0,1]$. By this algebraically demanding process, a discrete time iterative map is achieved for the system of Eq. (1). This iterative formula describes the cyclic operation between the two circuit topologies, which repeat themselves (on and off states) according to the equivalent duty cycle signal described above. A more representative way of the mathematical process is given in the Appendix but the details of an extensive description of the iterative map derivation process are explained in [16]. Although this is a simple mathematical model, this repetition of the different circuit topologies is the main responsible factor that destroys the smoothness of the dynamics and causes nonlinear phenomena [17].

\section{Switching Frequency Impact in Steady State}

As mentioned in the beginning, switching frequency is an important factor for every converter. Figure 3 shows the result of inductor's current in the steady state. The switching harmonics are multiples of the switching frequency with decaying amplitude as the order of switching frequency harmonic increases. The same behavior is observed in the experiment, too. It is also observed that, as the switching frequency increases, the current harmonics become smaller. They can be easily attenuated since high frequency harmonics require smaller filters. The whole idea is presented in Fig. 4. The vertical axis is the ratio of the biggest switching harmonic over the fundamental one. It is obvious that under this kind of operation the low switching frequencies offer big amplitude harmonics. Therefore, it will be of great importance if these harmonics could be spread to higher wideband frequencies with lower amplitudes. It is worth mentioning here, that in the experimental results for switching frequencies higher of $80 \mathrm{kHz}$ prototype Silicon Carbide TMosfets are used since Silicon devices are not capable to operate in these higher frequency regions.

In addition, the duty cycle of the converter $(d)$ follows the steady state duty cycle $(D)$ with more accuracy when 


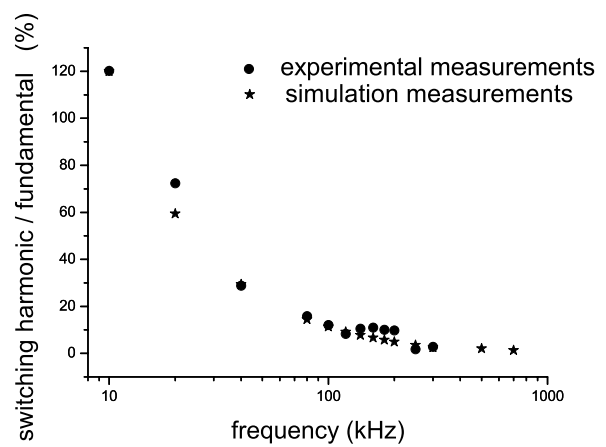

Fig. 4 Switching harmonics are getting smaller as switching frequency increases during steady state.

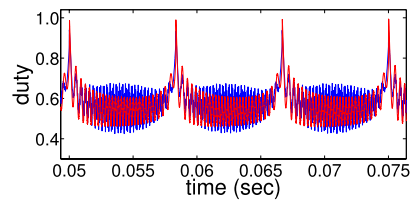

(a) simulation

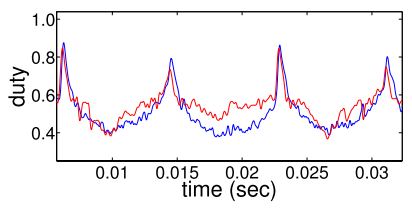

(b) experiment
Fig. 5 Duty cycle (d) follows the steady state duty cycle (D) better in higher switching frequencies. Blue graph is for $15 \mathrm{kHz}$ and the red one is for $50 \mathrm{kHz}$.

the switching frequency increases. In Fig. 5 the duty cycle is presented for two switching frequencies in the simulation and the experiment. It is clear that the deviation from the steady state duty cycle becomes smaller as the switching frequency increases. In detail, the steady state duty cycle was set to $D=0.6$. The figure shows that at the lower switching frequency the duty cycle $d$ follows $D$ in a wider value range.

In this section, we summarized the advantages of the classical steady state of AC/AC converter. Instead, the disadvantages of this operation should be the motive for further research and how they can be avoided with the help of nonlinear dynamics. In the next section, the chaotic operation will be proven numerically and how this operation can boost the converter performance eliminating the prementioned disadvantages.

\section{Test for Chaos and Performance Improvement}

In this section, the chaotic behavior will be qualitatively and quantitatively described. This behavior appears in particular with high feedback gain operation which is desired in order to make sure that the output signal will follow the reference signal as precisely as possible [18]. Figure 6 shows the dependence of one state variable versus the controllable parameter, which is the gain of the feedback control. Suppose that the steady state signal oscillates with the fundamental frequency within the interval $[-1,1]$. Each measurement in the bifurcation diagram is taken for every gain value from the equivalent time series around a very small time interval in the $n+1$ switching cycle when the transient response remains until the $n$th cycle. In this data acquisition this small time interval corresponds to the condition that the signal has

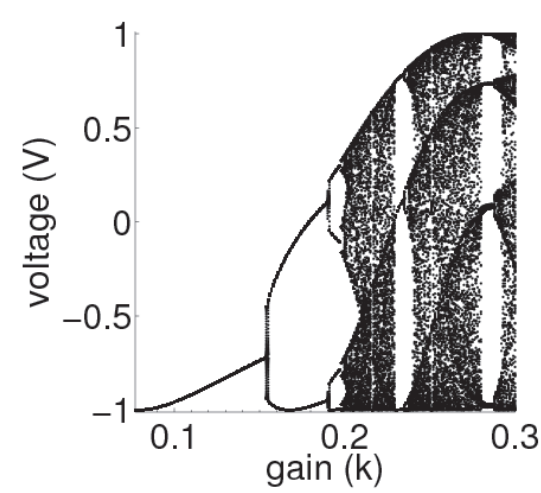

Fig. 6 The dependence on the controllable parameter is qualitatively described by its bifurcation diagram.
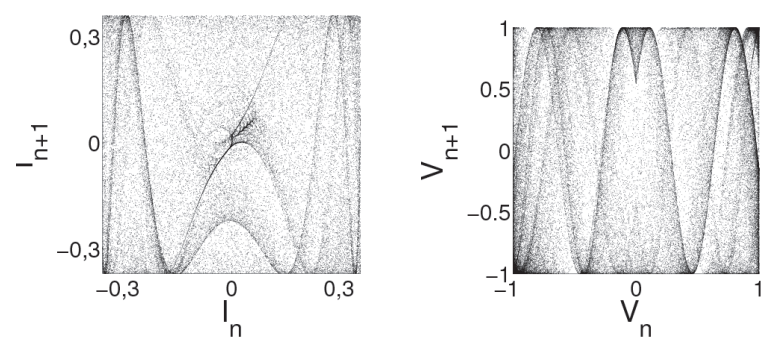

Fig. 7 Iterative plots for current and voltage during the chaotic operation. The predominant frequencies form distinctive lines among the chaotic data.

the maximum negative value -1 . For each gain value we gather the data at the peak. In the steady state all the data are almost near to -1 . This can be seen in the bifurcation diagram for the low gain values. As the diagram shows, increasing the gain value brings a period- 1 solution until gain $k \approx 1.53 \mathrm{E}-1$. Then a series of period doubling bifurcations undergoes until gain reaches the value of around $2 \mathrm{E}-1$ where 4 chaotic bands exist which are merging into two and finally into one chaotic band bounded to the maximum limits. In fact, these bifurcations happen because of the topological sequence change in the converter. In every switching converter boundaries exist between continuous and discontinuous modes of operation as mentioned in [16]. In other words these non-smooth border-collision bifurcations occur because of the circuit structural alteration in every switching cycle. Moreover, some windows of period-3 and period-4 periodicity are obvious. Some bifurcation veils are also visible declaring the regions where the orbits are most probable. By this diagram, the mechanism that drives the operation of the converter from period- 1 to multiple period operation to chaotic operation is isolated and described. In other words, here, the dynamic system's dependence on the controllable parameter is qualitatively demonstrated. It should be mentioned that the RLC parameters used for the simulations are $50 \Omega, 0.45 \mathrm{mH}$, and $33 \mu \mathrm{F}$ respectively.

In Fig. 7 the iterative plots of current and voltage are shown during these chaotic oscillations. The iterative plots cover all the area because of the random oscillations. They are unable to be connected into an orbit that closes itself 


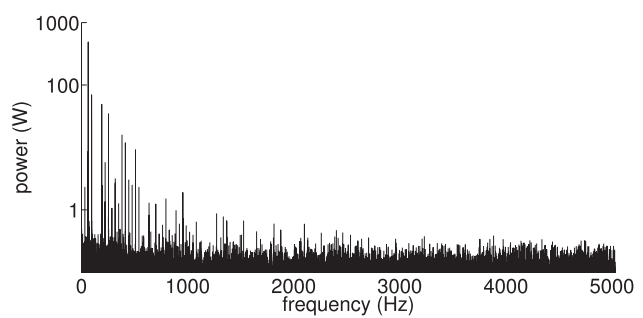

Fig. 8 Responsible frequency for the power flow is mainly the fundamental one. Even the following biggest harmonic has five times smaller amplitude than the fundamental.

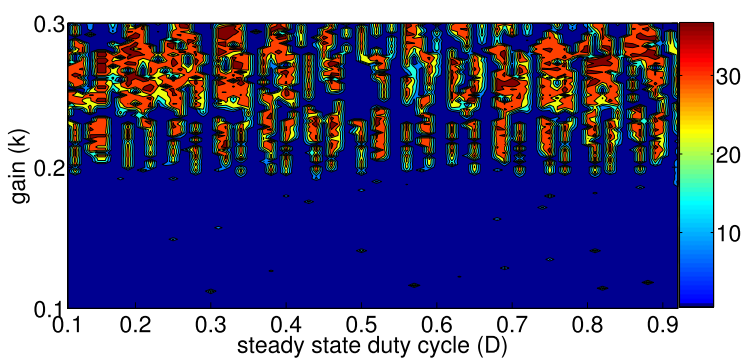

Fig. 9 Nonlinearity effect into the parameter space. The most blue area is period-1 solution while the brown one is chaotic. Even at high gains, period-1 solution can be succeeded by fine-tuning $\mathrm{D}$. These areas form stable period-1 'rivers' between the 'islands' of chaotic operation.

but because of the predominant frequencies specific lines can be distinguished through these graphs. These lines are formed mainly because of the fundamental frequency and this is proven by the power spectrum at Fig. 8. It is clear that the level of wide-band noise is minimum compared to the fundamental and even the biggest harmonic is around five times smaller than the fundamental. Thus, an operation during which the fundamental frequency is the responsible one for the power flow is succeeded.

Steady state duty cycle $(D)$ is also a parameter which governs the change of steady state. Converters usually change their duty cycle when load is changing, some transient effect takes place etc. In Fig. 9 the different dynamical modes of the operation are geometrically plotted in the parameter space $D$ and $k$. The 'nonlinearity density' is presented via the coloring. It starts from the blue area which denotes the stable period- 1 solution and goes until the brown one which is the chaotic region. The number of possible states is depicted via the discrete color-map. The middle stages, the period doubling process, are not very clear since as we described before this is just a qualitative separation of the nonlinear regions. In detail, for every pair of $(D, k)$ the simulation result is saved. Then, the higher number of periods the signal contains the closer to the brown color the equivalent area will be. Therefore, the most chaotic regions will have the brown color and the period-1 solution will have the blue one. As a result this 'nonlinearity density' can be determined. As it was expected in the low gain regions there are stable period operations and at high gains the complexity of the operation is increased dramatically. Another interesting observation is that although the high gain causes chaotic

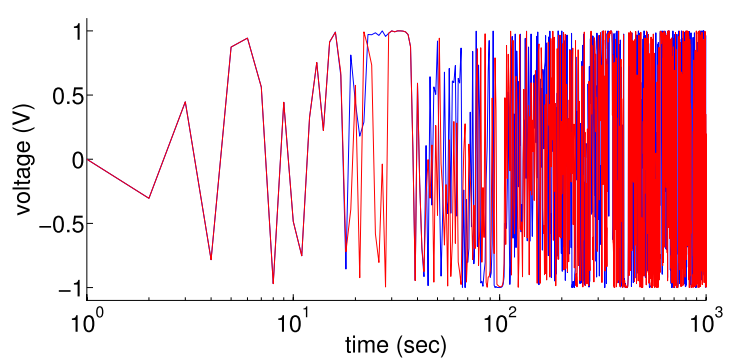

Fig. 10 Voltage time series for two very close initial conditions. After a short period of time the two trajectories are completely different.

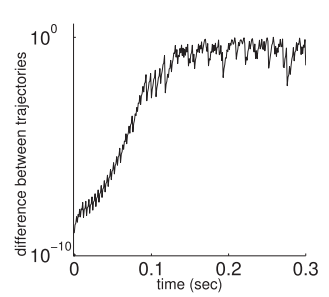

(a) divergence

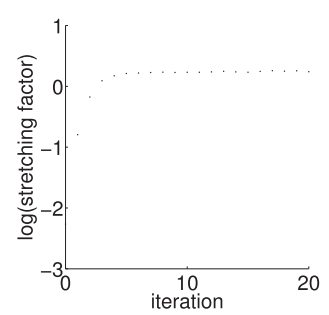

(b) LLE
Fig. 11 Left the exponential divergence from two initial conditions and right the calculation for the largest Lyapunov exponent. It can be found from the slope in the beginning of the figure.

operation there are certain areas, let's call them here 'rivers', where stable period operation can be succeeded. Even if the gain has to remain in high values, there is still possibility of stable operation along these 'rivers', meaning by fine adjustment of the steady state duty cycle. Figure 9 is a conspicuous description of the complex impact nonlinear dynamics can generate. Fast and accurate control can switch appropriately to the optimized operation by moving along into the parameter space.

One other test is the sensitiveness to the initial conditions. In Fig. 10 two time series are plotted together. The blue one is for zero initial conditions and the red one is for slightly bigger ones (1E-7). The horizontal axis has a logarithmic scale in order to show the behavior more clearly. In the beginning, the two trajectories exactly follow the same path, soon one of them seems to be a bit faster. Finally, it is obvious that the trajectories are completely different, always bounded to the limits of the oscillation. In Fig. 11 the divergence growth between those two trajectories is depicted on the left part of the figure. It can be noticed that the difference between the two orbits never converges to minimum again. The two time series, even bounded to the same limits, they are completely different. The right part of Fig. 11 is the calculation of the largest Lyapunov exponent (LLE) of these chaotic time series. The first method to calculate the LLE is the Rosenstein algorithm [19] which is based on the reconstruction of the attractor dynamics from a single time series by embedding this time series into its embedded dimensional phase space, lagging a specific amount of time units. Only from the first few points we can take the LLE estimation since as the algorithm continues the data reconverge and the slope falls to zero. If the calculated slope is 
estimated in a least square sense the result for the LLE is 0.547 . In order to verify more whether the result is chaotic or not the TISEAN [20] toolbox is used as well, giving a result of the LLE at 0.576. Although the two numbers are not exactly the same since different algorithms and procedures were implemented, it is verified that the LLE is a positive number giving the quantitative characteristics of our chaotic time series.

The practical side of all the above is that a converter can work in this chaotic region. With the proper control it will have a stable state and also the power flow will be concentrated to the fundamental frequency. In other words the harmonic content will be at minimum. This will bring great advantages especially when the switching frequency should remain at low levels since according to Fig. 4 with the classical approach, the low switching frequencies bring great amount of harmonics. Instead, chaotic operation along with suitable control could fix the prementioned problem. Therefore, a wider range of operation arises for fast-response and more agile power $\mathrm{AC} / \mathrm{AC}$ converters.

\section{Experimental Results and Discussion}

In Fig. 12 experimental phase portraits are presented. In Fig. 12(a) the circle depicts the base frequency (fundamental) and the curly behavior is caused by the switching impact. RLC parameters are the same as in simulations. The data have been averaged in order to have a better view but there is a small loss of amplitude information. The feedback gain was set at 0.1. In Fig. 12(b) there is a small extra circle in the phase portrait when the gain was increased at 0.13 . From the data analysis it is found that there is also the 2nd harmonic except for the fundamental and switching harmonics. Thus, the converter is driven to a period-2 operation. Moreover at $k=0.17$ a period- 3 operation appears as it is shown in Fig. 12(c). The graph for the current against the derivative of the current is also plotted in Fig. 12(d) in order to show more clearly this operation. There is a small

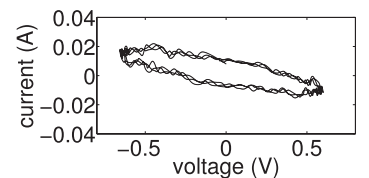

(a)

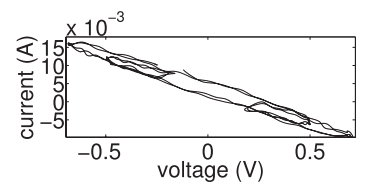

(c)

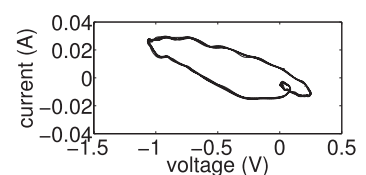

(b)

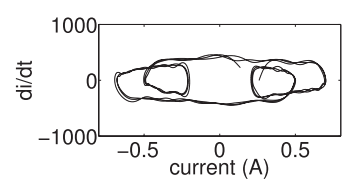

(d)
Fig. 12 Experimental phase portraits. Steady state is observed in (a) with the main circle representing the base line frequency and the curly behavior because of the switching frequency. Period- 2 solution at (b) and period- 3 at (c). (d) shows clearly the period-3 by plotting the current versus its derivative. Period-3 has higher order harmonics and a reduced overall amplitude than period-2. discrepancy with the simulation results which may be due to the fact that in the simulations parasitic effects and resistances from the semiconductors haven't been taken under consideration.

Two important phase portraits are shown in Fig. 13. They were succeeded in higher gain regions and it was noticed that semi-periodic random oscillations occurred. The left subfigure presents bilateral random oscillations in unexpected moments while in the right one there is a specific time in every cycle during which there is a different period every time and therefore it makes a unilateral semiperiodicity. This might happen because of grazing phenomena [18] driving the operation to a multi-period solution. It is worth noting that in the bilateral solution, duty cycle saturation was noticed fact that origins from nonlinearity. This duty cycle saturating nonlinearity was observed in the simulations, too. This fact ensures the border collision case since such kind of nonlinearity is also one of the basic causes for border collision bifurcations.

At period 2 and 3 operations it is the lower harmonics that cause this behavior. In the chaotic oscillations of the numerical results there is a broadband spectrum. In other words there are many frequencies contained in the signal. In [14] there is a comparison of a comb-like spectrum and a broadband chaotic spectrum. It was concluded that the broadband spectrum brought a significant reduction of harmonics in the converter operation.

In our work, period- 2 operation contains the 2 nd harmonic with $52.7 \%$ the amplitude of the fundamental, 3rd one with $14.7 \%$, 4th one with $11.6 \%$ and 5th one with $7.6 \%$. These numbers along with period- 3 orbit and the semi-periodic solutions are summarized in Table 1. In just one higher order of period-n operation, an amplitude reduction of the harmonics is achieved and also the main harmonics are shifted to higher orders. If the inverses of harmonic orders are used as weights a $64.22 \%$ harmonic reduction is estimated between period 2 and 3 operations. The unilateral semi-periodic solution appears a reduction of harmonics at $68.69 \%$ and the bilateral semi-periodic one is at $32.44 \%$ compared with period-2 solution. The double-sided semiperiodic one has an evenly distributed harmonic spectrum and only the third harmonic is quite big. If this main har-
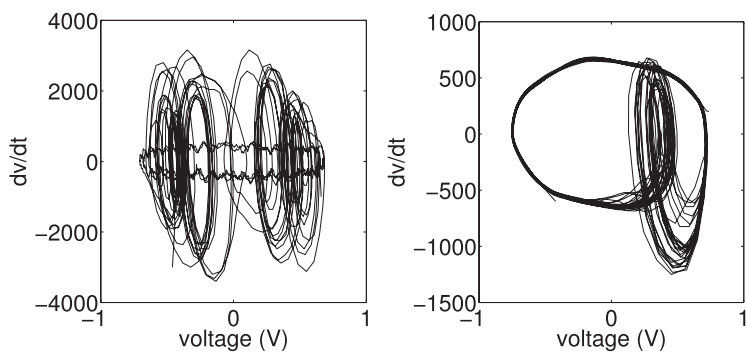

Fig. 13 Semi-periodic random oscillations in experiment. The right one is called unilateral semi-periodic solution because the randomness happens in one side of the main circle path when the gain $k$ was set at 0.25 . The left one is called bilateral (or multilateral) because the extra periods are all over the fundamental circle $(k=0.3)$. 
Table 1 Harmonics amplitudes during period 2 and 3 (\% of the fundamental). Higher order semi-periodic solutions were also observed (unilateral and bilateral). At higher periods, shifting to higher harmonic orders and lower amplitudes is expected. The semi-periodic random solutions have smaller harmonic amplitudes except the third harmonic in the bilateral solution.

\begin{tabular}{ccccc}
\hline \hline Period No. & 2nd & 3rd & 4th & 5th \\
\hline 2 & $52.7 \%$ & $14.7 \%$ & $11.6 \%$ & $7.6 \%$ \\
3 & $0 \%$ & $43 \%$ & $0 \%$ & $10 \%$ \\
unilateral & $13.4 \%$ & $11.1 \%$ & $9.1 \%$ & $8.1 \%$ \\
bilateral & $9 \%$ & $65.5 \%$ & $11.1 \%$ & $8.7 \%$ \\
\hline \hline
\end{tabular}
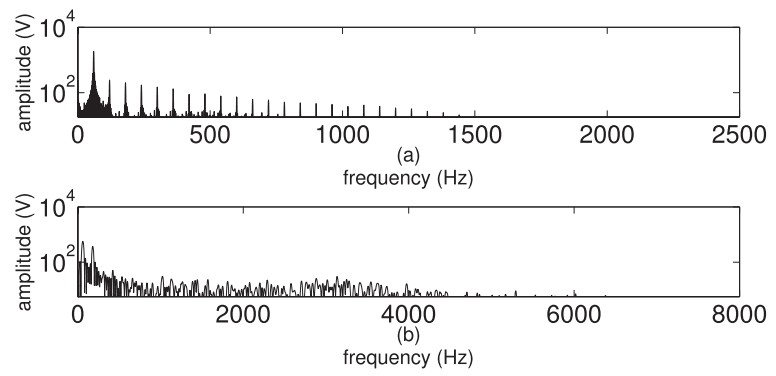

Fig. 14 (a) Unilateral solution spectrum and (b) bilateral solution spectrum. In the bilateral solution there is a more wide and without spikes spectrum. Every harmonic greater than $1 \%$ of the fundamental amplitude is shown.

monic is not considered, like in 3-phase circuits, the sum of all the others is estimated less than $10 \%$ of harmonic contribution compared always with the period- 2 solution. Therefore, there is a tendency of suppressing the low order and switching harmonics into a wide frequency area. Another approach to understand more the semi-periodic solutions is the frequency spectrums presented in Fig. 14. Figure 14(a) is for the unilateral solution and (b) for the bilateral one. The limits for the vertical axis have been set in such a way that only the greater harmonics of $1 \%$ of fundamental amplitude are shown. It is obvious that in the bilateral solution spectrum there is a much more broadband spectrum than the unilateral one and it is smoother, too. A final remark of the random oscillations is about their total harmonic distortion (THD). The unilateral solution has a THD equal to $4.9 \%$ while the bilateral's one is $4.3 \%$ without taking under consideration the third harmonic. These values meet the overall standards set by IEEE std. 519 - 1992. Applications and simple examples of these standards can be found in [21].

The problems of analogue approach made the period doubling operation data acquisition more difficult. Extreme caution must be paid also to the controllable parameters since as it was described in the previous section, the outcome varies significantly with minor parameter changes. For example, digital control allows accurate and high precision parameter choice, therefore moving along the various modes of Fig. 9, which are neighboring close to each other, will be an easier task. Nonlinear phenomena in AC/AC conversion are quite a new research area. Chaos and other instabilities especially in matrix converters can also be found in [22], [23].

\section{Conclusion}

The switching dynamics were studied through the derivation of a discrete time iterative map for the sake of revealing the possible operational regimes in the AC/AC conversion. Periodic and chaotic wide range frequency oscillations were observed. The chaotic operation was confirmed through qualitative and quantitative procedures based on bifurcation diagrams and Lyapunov exponents, respectively.

The most important goal is to spread the switching impact at higher wide range frequencies, because on one hand it is easier to attenuate them and on the other hand because of the EMC regulations, which require broadband noise. Based on the numerical results it was proven that the power flow was mainly because of the fundamental line frequency. In the experiments multi-period random oscillations were also observed, confirming the numerical analysis. Therefore, there exists the possibility for a practical application of chaotic operated AC/AC converter design.

A fundamental proportional gain feedback control was used in order to investigate the dynamics behavior and also, the impact of high switching frequency was observed in the steady state. The filter current harmonics were getting of smaller amplitude as the switching frequency increased. High gain is obligatory in order to confirm a fast and agile converter operation but contrarily high gain brings great abnormalities due to nonlinearity and that was the main topic covered in this paper.

Future work should include the study of the behavior on transient state and also the ability to control the chaotic regimes by driving them to stable operations. In other words, operating under specific control mechanisms, the converter could switch easily and faster to other frequencies since the chaotic regimes contain many periodic states, thus a multi-frequency converter could also be succeeded.

\section{Acknowledgments}

This work was partially supported by the Regional Innovation Cluster Program "Kyoto Environmental Nanotechnology Cluster" and the Excellent Graduate Schools Program in Kyoto University. We would also like to acknowledge the assistance of ROHM Semiconductor Co. Ltd. for the prototype Silicon Carbide semiconductor providing.

One of the authors (A.K.) would like to dedicate this work to the memory of V. Kordonis (10.10.192028.11.2012), a beloved grandfather and master of his craft.

\section{References}

[1] D.C. Hamill, "Power electronics: A field rich in nonlinear dynamics," Proc. 3rd Int. Specialists Workshop on Nonlinear Dynamics of Electronic Systems NDES'95, Citeseer, 1995.

[2] H.S. Patel and R.G. Hoft, "Generalized techniques of harmonic elimination and voltage control in thyristor inverters: Part $\mathrm{i}-$ harmonic elimination,” IEEE Trans. Ind. Appl., no.3, pp.310-317, 1973. 
[3] H.S. Patel and R.G. Hoft, "Generalized techniques of harmonic elimination and voltage control in thyristor inverters: Part iivoltage control techniques," IEEE Trans. Ind. Appl., no.5, pp.666673, 1974.

[4] G.H. Choe, A.K. Wallace, and M.H. Park, "An improved pwm technique for AC choppers," IEEE Trans. Power Electron., vol.4, no.4, pp.496-505, 1989.

[5] K.E. Addoweesh and A.L. Mohamadein, "Microprocessor based harmonic elimination in chopper type AC voltage regulators," IEEE Trans. Power Electron., vol.5, no.2, pp.191-200, 1990.

[6] A.M. Trzynadlowski, Introduction to modern power electronics, Wiley, 2010.

[7] J.M. Rivas, D. Jackson, O. Leitermann, A.D. Sagneri, Y. Han, and D.J. Perreault, "Design considerations for very high frequency DCDC converters," Power Electronics Specialists Conference, 2006. PESC'06. 37th IEEE, pp.1-11, 2006.

[8] H. Wang, F. Wang, and J. Zhang, "Power semiconductor device figure of merit for high-power-density converter design applications," IEEE Trans. Electron Devices, vol.55, no.1, pp.466-470, 2008.

[9] J.W. Kolar, T. Friedli, J. Rodriguez, and P.W. Wheeler, "Review of three-phase pwm AC-AC converter topologies," IEEE Trans. Ind. Electron., vol.58, no.11, pp.4988-5006, 2011.

[10] J. Rodriguez, M. Rivera, J.W. Kolar, and P.W. Wheeler, "A review of control and modulation methods for matrix converters," IEEE Trans. Ind. Electron., vol.59, no.1, pp.58-70, 2012.

[11] Y.M. Lai, S.C. Tan, and S.N. Chan, "A fast response zero-voltagetransition pulse-width modulation boost converter using a variable gain control technique," IEEE International Conference on Industrial Technology, 2008. ICIT 2008. pp.1-6, 2008.

[12] F. Kurokawa and S. Higuchi, "A novel digital variable integral gain control for DC-DC converter," 15th International Conference on Electrical Machines and Systems (ICEMS), 2012 pp.1-5, 2012.

[13] G. Mazzini, R. Rovatti, and G. Setti, "Chaos-based asynchronous DS-CDMA systems and enhanced rake receivers: Measuring the improvements," IEEE Trans. Circuits Syst. I, Fundam. Theory Appl., vol.48, no.12, pp.1445-1453, 2001.

[14] D.C. Hamill, J.H. Deane, and P.J. Aston, "Some applications of chaos in power converters," Update on New Power Electronic Techniques (Digest No: 1997/091), IEE Colloquium on, pp.5-1, IET, 1997.

[15] Z. Suto, I. Nagy, and E. Masada, "Avoiding chaotic processes in current control of AC drive," Power Electronics Specialists Conference, 1998. PESC 98 Record. 29th Annual IEEE, pp.255-261, 1998.

[16] C.K. Tse, Complex behavior of switching power converters, CRC Press, 2003.

[17] R.M. May, et al., "Simple mathematical models with very complicated dynamics," Nature, vol.261, no.5560, pp.459-467, 1976.

[18] Z.T. Zhusubaliyev, E. Mosekilde, A.I. Andriyanov, and G.Y. Mikhal'chenko, "High-feedback operation of power electronic converters," Electronics, vol.2, no.2, pp.113-167, 2013.

[19] M.T. Rosenstein, J.J. Collins, and C.J. De Luca, "A practical method for calculating largest Lyapunov exponents from small data sets," Physica D: Nonlinear Phenomena, vol.65, no.1, pp.117-134, 1993.

[20] R. Hegger, H. Kantz, and T. Schreiber, "Practical implementation of nonlinear time series methods: The tisean package," Chaos: An Interdisciplinary Journal of Nonlinear Science, vol.9, no.2, pp.413435, 1999.

[21] T.M. Blooming and D.J. Carnovale, "Application of IEEE std 5191992 harmonic limits,” Pulp and Paper Industry Technical Conference, 2006. Conference Record of Annual, pp.1-9, 2006.

[22] C. Xia, P. Song, T. Shi, and Y. Yan, "Chaotic dynamics characteristic analysis for matrix converter," IEEE Trans. Ind. Electron., vol.60, no.1, pp.78-87, 2013.

[23] S.M. Cox and J.C. Clare, "Nonlinear development of matrixconverter instabilities," J. Engineering Mathematics, vol.67, no.3, pp.241-259, 2010.

\section{Appendix: Iterative Map Details}

More information will be given about the derivation of the iterative map in a more representative way. The switching variable $s$ takes two values in Eq. (1):

- $s=0$ :

This is called off state. During this state, $\mathrm{SW}_{1}$ is off and $\mathrm{SW}_{2}$ is on. After solving the system of equations, for the $\left[t_{n}, t_{n^{\prime}}\right)$ interval, the result for the current and voltage will be:

$$
\left\{\begin{array}{l}
i_{1}(t)=f_{1}\left(u\left(t_{n}\right), i\left(t_{n}\right),\left(t-t_{n}\right)\right) \\
u_{1}(t)=f_{2}\left(u\left(t_{n}\right), i\left(t_{n}\right),\left(t-t_{n}\right)\right)
\end{array}\right.
$$

Now we set in the above equations:

$t=t_{n^{\prime}}$

$t_{n^{\prime}}-t_{n}=(1-d) T$

and the result becomes:

$$
\left\{\begin{array}{l}
i_{1}\left(t_{n^{\prime}}\right)=f_{1}\left(u\left(t_{n}\right), i\left(t_{n}\right),((1-d) T)\right) \\
u_{1}\left(t_{n^{\prime}}\right)=f_{2}\left(u\left(t_{n}\right), i\left(t_{n}\right),((1-d) T)\right)
\end{array}\right.
$$

- $s=1$ :

This is called on state. During this state, $\mathrm{SW}_{1}$ is on and $\mathrm{SW}_{2}$ is off. The system of equations, for the $\left[t_{n^{\prime}}, t_{n+1}\right)$ interval, results in:

$$
\left\{\begin{array}{l}
i_{2}(t)=g_{1}\left(u\left(t_{n^{\prime}}\right), i\left(t_{n^{\prime}}\right),\left(t-t_{n^{\prime}}\right)\right) \\
u_{2}(t)=g_{2}\left(u\left(t_{n^{\prime}}\right), i\left(t_{n^{\prime}}\right),\left(t-t_{n^{\prime}}\right)\right)
\end{array}\right.
$$

Now we set in the above equations:

$t=t_{n+1}$

$t_{n+1}-t_{n^{\prime}}=d T$

and the result becomes:

$$
\left\{\begin{array}{l}
i_{2}\left(t_{n+1}\right)=g_{1}\left(u\left(t_{n^{\prime}}\right), i\left(t_{n^{\prime}}\right),(d T)\right) \\
u_{2}\left(t_{n+1}\right)=g_{2}\left(u\left(t_{n^{\prime}}\right), i\left(t_{n^{\prime}}\right),(d T)\right)
\end{array}\right.
$$

The arbitrary switching time $t_{n^{\prime}}$ is undesirable. Therefore, using Eq. (A.2) $t_{n^{\prime}}$ can be eliminated in Eq. (A.4) and as a result the difference equation of the iterative map is created:

$$
\left\{\begin{array}{l}
i\left(t_{n+1}\right)=q_{1}\left(u\left(t_{n}\right), i\left(t_{n}\right), d\right) \\
u\left(t_{n+1}\right)=q_{2}\left(u\left(t_{n}\right), i\left(t_{n}\right), d\right)
\end{array}\right.
$$

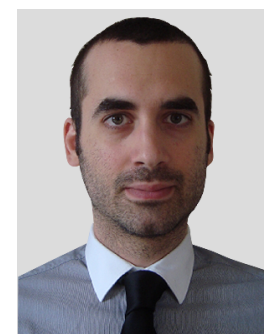

Alexandros Kordonis was born in Greece on July 7, 1985. He received his diploma of Electrical and Computer Engineering from $\mathrm{Na}-$ tional Technical University of Athens in Greece, 2009. He is currently working towards his Ph.D degree in the Department of Electrical Engineering at Kyoto University. His main research interests are power conversion, nonlinear dynamics, and control systems. 


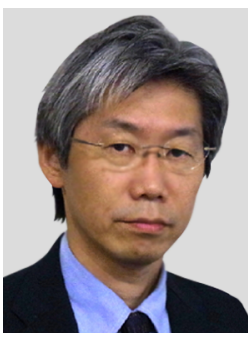

Takashi Hikihara received his Ph.D. degree from Kyoto University, in 1990. In 1987 he joined Kansai University. From 1993 to 1994, he was a visiting researcher at Cornell University. Since 1997 he has been with Department of Electrical Engineering at Kyoto University, where he is currently a Professor. His research interests are including structural analysis of nonlinear system applications and control of nonlinear dynamics, nano-mechanical systems, and power electronics. He is a member of IEEE,

APS, SIAM, SICE, ISICE and IEE, Japan. 